\title{
THE PRESSURE FIELD IN THE GAS-LUBRICATED STEP SLIDER BEARING
}

\author{
I. PENESIS ${ }^{1}$, J. J. SHEPHERD ${ }^{2}$ and H. J. CONNELL ${ }^{2}$
}

(Received 5 February, 2002; revised 27 August, 2003)

\begin{abstract}
Singular perturbation methods are applied to an analysis of the operation of an isothermal gas step slider bearing of narrow geometry and operating at moderate bearing numbers. Approximate expressions are obtained for the pressure field in the lubricating gap, as well as the load-carrying capacity of the bearing; and the influence of the nature of the bearing step on those quantities is investigated. Comparisons are made with results obtained using a standard numerical package.
\end{abstract}

\section{Introduction}

A fundamental problem arising in the performance analysis of a gas-lubricated bearing is that of determining the steady-state pressure distribution in the bearing, its loadcarrying capacity, and possibly other physically relevant design characteristics. For a bearing operating isothermally, the nonlinearity of the Reynolds equation determining the pressure field rules out the likelihood of obtaining closed-form analytic solutions, except in particular cases of very simple geometry. Consequently, in most situations, other (appropriate) methods must be resorted to.

Often, a small parameter is associated with the problem-either arising from the physical operating conditions of the bearing, as in DiPrima [2], or Schmitt and DiPrima [6], or from the bearing geometry, as in Shepherd and DiPrima [7] or Penesis, Shepherd and Connell $[4,5]$ - perturbation techniques may be applied to obtain a closed asymptotic expression that approximates the pressure in some suitable sense. This may also be used to construct expressions to approximate the load, and other quantities. It should be noted that although such methods limit the results to a particular

\footnotetext{
${ }^{1}$ Faculty of Maritime Transport and Engineering, Australian Maritime College, Launceston, Australia; e-mail: i.penesis@mte.amc.edu.au.

${ }^{2}$ Department of Mathematics, RMIT University, Melbourne, Australia; e-mail: jshep@rmit.edu.au.

(C) Australian Mathematical Society 2004, Serial-fee code 1446-1811/04
} 
range of parameter values, they are advantageous in that they generate closed-form expressions applicable over a general range of values of any other parameters relevant to the problem. Thus they have an advantage over numerical calculations that can only cover a range of parameter values at the expense of numerous recalculations. Moreover, they are often applicable in just those parameter ranges where numerical techniques experience instabilities.

In the present paper, we consider the situation of a narrow isothermal gas slider operating at moderate speed. Thus the identifiable small parameter is the breadth parameter (the ratio of width to length). In terms of this (small) parameter, the perturbation problem determining the pressure field is known to be singular, so that appropriate methods - the method of matched expansions here-are applied to obtain an approximation to the pressure over the whole bearing area. As an added complication, we assume that the bearing profile function displays a finite discontinuity ('step') transverse to the direction of travel, and is smooth elsewhere. To deal with this, we adopt the approach of [4], and construct approximations on the smooth sub-domains, joining them by appropriate conditions at the step. This composite approximation is then used to approximate the load-bearing capacity of the whole bearing.

The complexity of the calculations limits our results here to the case of the so-called 'wedge' bearing, where the profile only varies in the travel direction, and is constant transversely. These results are found to compare very favourably with finite element calculations using a standard commercial package.

\section{Governing equations}

The geometry of the step slider bearing considered here is as displayed in Figure 1. The lower surface (the $X Z$ plane here) moves with constant speed $U_{0}$ in the positive $X$-direction, while the (stationary) upper surface represented by $Y=H(X, Z)$ has a single finite transverse jump discontinuity along the line $X=x_{0} L$, for some dimensionless $0<x_{0}<1$.

For the case of isothermal flow, the pressure in the bearing gap may be shown to be a solution of the nonlinear Reynolds equation which becomes, in dimensionless form,

$$
\epsilon^{2} \frac{\partial}{\partial x}\left(h^{3} p \frac{\partial p}{\partial x}\right)+\frac{\partial}{\partial z}\left(h^{3} p \frac{\partial p}{\partial z}\right)=\epsilon^{2} \Lambda \frac{\partial}{\partial x}(p h),
$$

where $p, x, z$ and $h$ are the dimensionless pressure, longitudinal variable, transverse variable and profile function scaled against the (constant) ambient pressure, $P_{a}$, length $L$, breadth $B$ and a representative value of $H, H_{0}$, respectively. Thus (2.1) is assumed to hold on $0 \leq x \leq 1,-1 / 2 \leq z \leq 1 / 2$. 


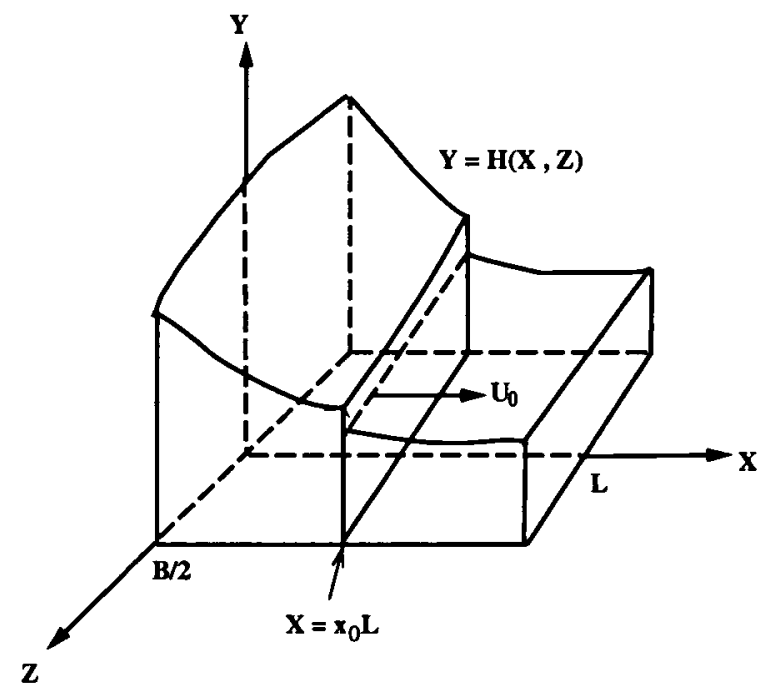

FIGURE 1. Geometry for the narrow gas step slider bearing.

The dimensionless parameters $\Lambda$ and $\epsilon$ are defined by

$$
\Lambda=\frac{6 \mu U_{0} L}{P_{a} H_{0}^{2}}, \quad \epsilon=\frac{B}{L}
$$

respectively. Here $\Lambda$, termed the bearing number, is a measure of flow speed, while $\epsilon$, the breadth parameter, gives the width to depth ratio of the bearing. Overall, the pressure $p$ depends on all of $x, z$ and the two parameters $\Lambda$ and $\epsilon$. However, since we are interested here in the case where $\epsilon$ is small (and $\Lambda=O(1)$ ), we will not display this $\Lambda$-dependence, and simply write the pressure $p$ as $p(x, z, \epsilon)$. Thus the condition that the pressure attain the ambient value $P_{a}$ at the bearing boundaries becomes

$$
p(x, \pm 1 / 2, \epsilon)=p(0, z, \epsilon)=p(1, z, \epsilon)=1
$$

for all $\epsilon>0$.

In what follows, we view the bearing surface as consisting of the union of two smooth bearing surfaces defined on the separate domains

$$
0 \leq x \leq x_{0},-1 / 2 \leq z \leq 1 / 2 \text { and } x_{0} \leq x \leq 1,-1 / 2 \leq z \leq 1 / 2 \text {, }
$$

and apply the basic techniques of [7] to these smooth sub-domains.

This approach requires us to solve (2.1) subject to appropriate boundary conditions at the edges of these sub-domains. While (2.3) holds at the exterior boundary of both 
regions, further conditions along the common boundary at $x=x_{0}$ are required. One of these is furnished by the stipulation that $p(x, z, \epsilon)$ be continuous there, that is,

$$
p\left(x_{0}-, z, \epsilon\right)=p\left(x_{0}+, z, \epsilon\right)
$$

for all $\epsilon>0,-1 / 2 \leq z \leq 1 / 2$. The other arises from the requirement that the mass flow across $x=x_{0}$ be continuous and is given mathematically by

$$
h^{3}(x, z) \frac{\partial p}{\partial x}-\left.\Lambda h(x, z)\right|_{x=x_{0}-}=h^{3}(x, z) \frac{\partial p}{\partial x}-\left.\Lambda h(x, z)\right|_{x=x_{0}+} .
$$

The condition (2.5) is readily obtained by integrating the partial differential equation (2.1) longitudinally across the step at $x=x_{0}$, and applying (2.4).

Note that (2.4) does not give the value of $p$ at $x=x_{0}$. We replace this condition by

$$
p\left(x_{0}-, z, \epsilon\right)=p\left(x_{0}+, z, \epsilon\right)=\lambda(z, \epsilon),
$$

where $\lambda(z, \epsilon)$ is a function to be determined in the subsequent calculations.

Thus, on the first domain, this pressure $p(x, z, \epsilon)$ is assumed to satisfy the partial differential equation (2.1), together with the boundary conditions

$$
p(x, \pm 1 / 2, \epsilon)=1, \quad 0 \leq x \leq x_{0},
$$

and

$$
\left.\begin{array}{c}
p(0, z, \epsilon)=1 \\
p\left(x_{0}, z, \epsilon\right)=\lambda(z, \epsilon)
\end{array}\right\} \quad-1 / 2 \leq z \leq 1 / 2 .
$$

On the second domain, the pressure satisfies (2.1), plus the boundary conditions

$$
p(x, \pm 1 / 2, \epsilon)=1, \quad x_{0} \leq x \leq 1,
$$

and

$$
\left.\begin{array}{c}
p\left(x_{0}, z, \epsilon\right)=\lambda(z, \epsilon) \\
p(1, z, \epsilon)=1
\end{array}\right\} \quad-1 / 2 \leq z \leq 1 / 2 .
$$

In the following sections, we apply perturbation methods based on $\epsilon \rightarrow 0$ to obtain representations for the pressure field in the leading and trailing sections of the bearing, and then, by applying the juncture conditions (2.4) and (2.5), construct the function $\lambda(z, \epsilon)$.

As we noted above, $\lambda(z, \epsilon)$, which represents the value of the pressure along the step discontinuity $x=x_{0}$, has now become one of the unknown quantities of the problem. Since we are to adopt a perturbation approach based on $\epsilon \rightarrow 0$, and since we might expect that for small $\epsilon$ (that is, in a narrow bearing) the pressure within the bearing would not deviate far from the ambient value, we propose that $\lambda(z, \epsilon)$ has the expansion

$$
\lambda(z, \epsilon)=1+\epsilon \lambda_{1}(z)+\epsilon^{2} \lambda_{2}(z)+\cdots,
$$

where $\lambda_{1}(z), \lambda_{2}(z), \ldots$ are to be determined. 


\section{Perturbation analysis in the leading section}

Here we consider the pressure field in the leading section $\left(0 \leq x \leq x_{0},-1 / 2 \leq\right.$ $z \leq 1 / 2)$ of the bearing, which is given as the solution of the boundary value problem (2.1), (2.7), (2.8). For small $\epsilon$, we propose an expansion of the form

$$
p(x, z, \epsilon)=\bar{p}_{0}(x, z)+\epsilon \bar{p}_{1}(x, z)+\epsilon^{2} \bar{p}_{2}(x, z)+\cdots,
$$

where barred quantities relate to the leading bearing section.

Substituting (3.1) into (2.1) and equating like powers of $\epsilon$ yields a sequence of differential equations for $\bar{p}_{0}, \bar{p}_{1}, \bar{p}_{2}, \ldots$ that are second order in $z$, and which may be solved to make (3.1) satisfy the boundary conditions on the boundaries $z= \pm 1 / 2$, $0 \leq x \leq x_{0}$. This gives

$$
\begin{aligned}
& \bar{p}_{0}(x, z) \equiv 1 \\
& \bar{p}_{1}(x, z) \equiv 0
\end{aligned}
$$

while

$$
\bar{p}_{2}(x, z)=\Lambda\left[F_{1}(x, z)-\frac{F_{1}(x, 1 / 2)}{F_{2}(x, 1 / 2)} F_{2}(x, z)\right]
$$

where

$$
F_{1}(x, z)=\int_{-1 / 2}^{z}\left(h^{-3}(x, s) \int_{0}^{s} \frac{\partial h}{\partial x}(x, t) d t\right) d s
$$

and

$$
F_{2}(x, z)=\int_{-1 / 2}^{z} h^{-3}(x, s) d s
$$

Thus the expansion (3.1) becomes

$$
p(x, z, \epsilon)=1+\epsilon^{2} \bar{p}_{2}(x, z)+O\left(\epsilon^{3}\right) .
$$

The expansion (3.3) is intended to represent the pressure throughout the whole of the leading section. However, it is clear that for arbitrary $\lambda_{1}(z), \lambda_{2}(z)$ it cannot meet the required boundary conditions at both edges $x=0$ and $x=x_{0}$. Thus this expansion represents the pressure on $0<x<x_{0},-1 / 2 \leq z \leq 1 / 2$ through terms of order $O\left(\epsilon^{2}\right)$, but fails in a neighbourhood of the (local) leading and trailing edges. Thus we expect boundary layer structure at the edges $x=0$ and $x=x_{0}$. Further, straightforward application of local analysis analogous to that of [7] shows that these layers are of thickness $O(\epsilon)$.

To analyse the pressure in the layer at $x=0$, we introduce the local variable $\bar{\xi}$, defined by $x=\epsilon \bar{\xi}$ where $\bar{\xi}=O(1)$ as $\epsilon \rightarrow 0$. Application of standard matching 
techniques [7] shows that the boundary layer expansion near $x=0$ (the leading edge of the leading section) takes the form

$$
\bar{P}_{L}(\bar{\xi}, z, \epsilon)=1+\epsilon^{2} \bar{P}_{L 2}(\bar{\xi}, z)+\cdots,
$$

where $\bar{P}_{L 2}$ is given by $\bar{P}_{L 2}(\bar{\xi}, z)=\bar{p}_{2}(0, z)+\bar{v}_{2}(\bar{\xi}, z)$, with $\bar{v}_{2}\left(\bar{\xi}_{,}, z\right)$ being given by the eigenfunction expansion

$$
\bar{v}_{2}(\bar{\xi}, z)=\sum_{n=1}^{\infty} c_{n} \phi_{n}(z) e^{-\bar{\xi} \sqrt{\lambda_{n}}}, \quad-1 / 2<z<1 / 2, \bar{\xi} \geq 0
$$

Here, the $\phi_{n}(z)$ are the eigenfunctions of

$$
\begin{aligned}
\frac{d}{d z}\left(h^{3}(0, z) \frac{d \phi_{n}}{d z}\right)+\lambda_{n} h^{3}(0, z) \phi_{n} & =0, \\
\phi_{n}( \pm 1 / 2) & =0,
\end{aligned}
$$

normalised with respect to the weight function $h^{3}(0, z) ; \lambda_{n}$ are the corresponding eigenvalues, while $c_{n}$ are the Fourier coefficients that make the expansion (3.1) meet the boundary conditions at $x=0$ (to the level in $\epsilon$ considered). Thus

$$
c_{n}=-\int_{-1 / 2}^{1 / 2} h^{3}(0, z) \bar{p}_{2}(0, z) \phi_{n}(z) d z, \quad n=1,2,3, \ldots
$$

In the present discussion, the subscript $L$ will denote quantities relating to the leading edge of the bearing section, while the subscript $T$ denotes those relevant to the trailing edge.

For the boundary layer near $x=x_{0}$, we introduce the local variable $\bar{\eta}=\left(x_{0}-x\right) / \epsilon$, $\bar{\eta}=O(1)$ and $0 \leq \bar{\eta} \leq \infty$, which, with $p\left(x_{0}-\epsilon \bar{\eta}, z, \epsilon\right) \equiv \bar{P}_{T}(\bar{\eta}, z, \epsilon)$, gives

$$
\frac{\partial}{\partial \bar{\eta}}\left(h^{3} \bar{P}_{T} \frac{\partial \bar{P}_{T}}{\partial \bar{\eta}}\right)+\frac{\partial}{\partial z}\left(h^{3} \bar{P}_{T} \frac{\partial \bar{P}_{T}}{\partial z}\right)=-\epsilon \Lambda \frac{\partial}{\partial \bar{\eta}}\left(\bar{P}_{T} h\right) .
$$

For a solution of (3.9) of the form

$$
\bar{P}_{T}(\bar{\eta}, z, \epsilon)=1+\epsilon \bar{P}_{T 1}(\bar{\eta}, z)+\epsilon^{2} \bar{P}_{T 2}(\bar{\eta}, z)+\cdots,
$$

we obtain, on substituting (3.10) into (3.9), and equating like powers of $\epsilon$,

$$
L_{0}^{-} \bar{P}_{T 1} \equiv \frac{\partial}{\partial \bar{\eta}}\left(h^{3}\left(x_{0}-, z\right) \frac{\partial \bar{P}_{T 1}}{\partial \bar{\eta}}\right)+\frac{\partial}{\partial z}\left(h^{3}\left(x_{0}-, z\right) \frac{\partial \bar{P}_{T 1}}{\partial z}\right)=0
$$


and

$$
\begin{aligned}
L_{0}^{-} \bar{P}_{T 2}= & -\frac{\partial}{\partial \bar{\eta}}\left(h^{3}\left(x_{0}-, z\right) \tilde{P}_{T 1} \frac{\partial \bar{P}_{T 1}}{\partial \bar{\eta}}-3 \bar{\eta} h^{2}\left(x_{0}-, z\right) \frac{\partial h}{\partial x}\left(x_{0}-, z\right) \frac{\partial \bar{P}_{T 1}}{\partial \bar{\eta}}\right) \\
& -\frac{\partial}{\partial z}\left(h^{3}\left(x_{0}-, z\right) \bar{P}_{T 1} \frac{\partial \bar{P}_{T 1}}{\partial z}-3 \bar{\eta} h^{2}\left(x_{0}-, z\right) \frac{\partial h}{\partial x}\left(x_{0}-, z\right) \frac{\partial \bar{P}_{T 1}}{\partial z}\right) \\
= & \Lambda \frac{\partial}{\partial \bar{\eta}}\left(h\left(x_{0}-, z\right) \bar{P}_{T 1}-\bar{\eta} \frac{\partial h}{\partial x}\left(x_{0}-, z\right)\right)
\end{aligned}
$$

where $h$ values are taken as $x \rightarrow x_{0}$ from the leading section.

The boundary conditions at $x=x_{0}, \bar{\eta}=0$ and at the edges $z= \pm 1 / 2$ are given by $\bar{P}_{T 1}(0, z)=\lambda_{1}(z), \bar{P}_{T 2}(0, z)=\lambda_{2}(z)$, for $-1 / 2 \leq z \leq 1 / 2$, and

$$
\bar{P}_{T 1}(\bar{\eta}, \pm 1 / 2)=\bar{P}_{T 2}(\bar{\eta}, \pm 1 / 2)=0,
$$

for $\bar{\eta} \geq 0$.

Since the equations for $\bar{P}_{T i}, i=1,2,3, \ldots$, are second order in $\bar{\eta}$, matching conditions yield the second $\bar{\eta}$ boundary conditions as

$$
\bar{P}_{T 1}(\bar{\eta}, z) \rightarrow 0, \quad \bar{P}_{T 2}(\bar{\eta}, z) \rightarrow \bar{p}_{2}\left(x_{0}-, z\right) \quad \text { as } \bar{\eta} \rightarrow \infty .
$$

The boundary value problem for $\bar{P}_{T 1}$ then becomes

$$
\begin{gathered}
L_{0}^{-} \bar{P}_{T 1}=0 \\
\bar{P}_{T 1}(0, z)=\lambda_{1}(z), \quad \bar{P}_{T 1}(\bar{\eta}, \pm 1 / 2)=0, \\
\bar{P}_{T 1}(\bar{\eta}, z) \rightarrow 0 \quad \text { as } \bar{\eta} \rightarrow \infty
\end{gathered}
$$

Solving (3.11)-(3.13), we obtain the solution

$$
\bar{P}_{T 1}(\bar{\eta}, z)=\sum_{n=1}^{\infty} j_{n} \chi_{n}(z) e^{-\bar{\eta} \sqrt{\kappa_{n}}}, \quad-1 / 2<z<1 / 2, \bar{\eta} \geq 0
$$

where

$$
j_{n}=\int_{-1 / 2}^{1 / 2} h^{3}\left(x_{0}-, z\right) \lambda_{1}(z) \chi_{n}(z) d z, \quad n=1,2,3, \ldots,
$$

are the Fourier coefficients, while $\kappa_{n}$ and $\chi_{n}(z)$ are the eigenvalues and eigenfunctions of the system

$$
\frac{d}{d z}\left(h^{3}\left(x_{0}-, z\right) \frac{d \chi_{n}}{d z}\right)+\kappa_{n} h^{3}\left(x_{0}-, z\right) \chi_{n}=0,
$$

with

$$
\chi_{n}( \pm 1 / 2)=0
$$


normalised with respect to the weight function $h^{3}\left(x_{0}-, z\right)$.

Note that the expression (3.14) for $\bar{P}_{T 1}$ relies on us knowing $\lambda_{1}(z)$-we have not, as yet, determined this quantity.

Now reconsider the partial differential equation for $\tilde{P}_{T 2}$, namely

$$
\begin{aligned}
L_{0}^{-} \bar{P}_{T 2}= & -\frac{\partial}{\partial \bar{\eta}}\left[h^{3}\left(x_{0}-, z\right) \bar{P}_{T 1} \frac{\partial \bar{P}_{T 1}}{\partial \bar{\eta}}-3 \bar{\eta} h^{2}\left(x_{0}-, z\right) \frac{\partial h}{\partial x}\left(x_{0}-, z\right) \frac{\partial \bar{P}_{T 1}}{\partial \bar{\eta}}\right] \\
& -\frac{\partial}{\partial z}\left[h^{3}\left(x_{0}-, z\right) \bar{P}_{T 1} \frac{\partial \bar{P}_{T 1}}{\partial z}-3 \bar{\eta} h^{2}\left(x_{0}-, z\right) \frac{\partial h}{\partial x}\left(x_{0}-, z\right) \frac{\partial \bar{P}_{T 1}}{\partial z}\right] \\
& +\Lambda\left(\frac{\partial}{\partial \bar{\eta}}\left(h\left(x_{0}-, z\right) \bar{P}_{T 1}\right)+\frac{\partial h}{\partial x}\left(x_{0}-, z\right)\right),
\end{aligned}
$$

together with the boundary conditions

$$
\begin{aligned}
& \bar{P}_{T 2}(0, z)=\lambda_{2}(z), \quad \bar{P}_{T 2}(\bar{\eta}, \pm 1 / 2)=0, \\
& \bar{P}_{T 2}(\bar{\eta}, z) \rightarrow \bar{p}_{2}\left(x_{0}-, z\right) \quad \text { as } \bar{\eta} \rightarrow \infty .
\end{aligned}
$$

If we define $\bar{w}_{2}(\bar{\eta}, z)$ by $\bar{P}_{T 2}(\bar{\eta}, z)=\bar{p}_{2}\left(x_{0}-, z\right)+\bar{w}_{2}(\bar{\eta}, z)$, (3.18) becomes

$$
\begin{aligned}
L_{0}^{-} \bar{w}_{2}= & -\frac{\partial}{\partial \bar{\eta}}\left[h^{3}\left(x_{0}-, z\right) \bar{P}_{T 1} \frac{\partial \bar{P}_{T 1}}{\partial \bar{\eta}}-3 \bar{\eta} h^{2}\left(x_{0}-, z\right) \frac{\partial h}{\partial x}\left(x_{0}-, z\right) \frac{\partial \bar{P}_{T 1}}{\partial \bar{\eta}}\right] \\
& -\frac{\partial}{\partial z}\left[h^{3}\left(x_{0}-, z\right) \bar{P}_{T 1} \frac{\partial \bar{P}_{T 1}}{\partial z}-3 \bar{\eta} h^{2}\left(x_{0}-, z\right) \frac{\partial h}{\partial x}\left(x_{0}-, z\right) \frac{\partial \bar{P}_{T 1}}{\partial z}\right] \\
& +\Lambda \frac{\partial}{\partial \bar{\eta}}\left(h\left(x_{0}-, z\right) \bar{P}_{T 1}\right),
\end{aligned}
$$

while the boundary conditions (3.19), (3.20) become

$$
\begin{aligned}
& \bar{w}_{2}(0, z)=\lambda_{2}(z)-\bar{p}_{2}\left(x_{0}-, z\right), \quad \bar{w}_{2}(\bar{\eta}, \pm 1 / 2)=0, \\
& \bar{w}_{2}(\bar{\eta}, z) \rightarrow 0 \quad \text { as } \bar{\eta} \rightarrow \infty .
\end{aligned}
$$

If we let $f(\bar{\eta}, z)$ denote the right-hand side of (3.21), while

$$
\bar{w}_{2}(\bar{\eta}, z)=\sum_{n=1}^{\infty} \bar{w}_{2 n}(\bar{\eta}) \chi_{n}(z)
$$

and

$$
\frac{f(\bar{\eta}, z)}{h^{3}\left(x_{0}-, z\right)}=\sum_{n=1}^{\infty} \gamma_{n} \chi_{n}(z)
$$

where the coefficients $\gamma_{n}$ are given by

$$
\gamma_{n}=\int_{-1 / 2}^{1 / 2} f(\bar{\eta}, z) \chi_{n}(z) d z, \quad n=1,2,3, \ldots
$$


we may apply the method of eigenfunction expansions [1] to show that the $\bar{w}_{2 n}$ are given as the solutions of the problems

$$
\begin{aligned}
\bar{w}_{2 n}^{\prime \prime}(\bar{\eta})-\kappa_{n} \bar{w}_{2 n}(\bar{\eta}) & =\gamma_{n}, \\
\bar{w}_{2 n}(0) & =a_{n}, \\
\bar{w}_{2 n}(\infty) & =0,
\end{aligned}
$$

for $n=1,2,3, \ldots$, where the initial values $a_{n}$ are given by

$$
a_{n}=\int_{-1 / 2}^{1 / 2} h^{3}\left(x_{0}-, z\right)\left[\lambda_{2}(z)-\bar{p}_{2}\left(x_{0}-, z\right)\right] \chi_{n}(z) d z, \quad n=1,2,3, \ldots
$$

Once all the quantities on the right-hand side $\kappa_{n}, \chi_{n}, \gamma_{n}, a_{n}$ are determined, we can then obtain an expression for $\bar{w}_{2}(\bar{\eta}, z)$. This requires us to find the functions $\lambda_{1}(z)$ and $\lambda_{2}(z)$, which appear in (3.15) and (3.29) respectively. However, it will turn out that this can only be done with useful results for special cases of $h$. These will be considered subsequently.

Combining expressions (3.3), (3.4) and (3.10) we obtain the expansion for the pressure

$$
\begin{aligned}
p(x, z, \epsilon)= & 1+\epsilon \bar{P}_{T 1}\left(\left(x_{0}-x\right) / \epsilon, z\right) \\
& +\epsilon^{2}\left[\bar{p}_{2}(x, z)+\bar{v}_{2}(x / \epsilon, z)+\bar{w}_{2}\left(\left(x_{0}-x\right) / \epsilon, z\right)\right]+O\left(\epsilon^{3}\right),
\end{aligned}
$$

on the first domain $0 \leq x \leq x_{0},-1 / 2 \leq z \leq 1 / 2$.

\section{Perturbation analysis in the trailing section}

The pressure in the trailing bearing section $\left(x_{0} \leq x \leq 1,-1 / 2 \leq z \leq 1 / 2\right)$ is given by the solution of the BVP comprising the differential equation (2.1) together with the boundary conditions (2.9), (2.10). The perturbation procedure to be adopted here is analogous to that of Section 3, with the only difference being that the function $\lambda(z, \epsilon)$ (still given by the expansion (2.11)) occurs in the boundary conditions at the leading edge of the trailing section, while the pressure reaches the ambient value at the trailing edge, $x=1$.

When calculations paralleling those of Section 3 are carried out, we arrive at the expansion for the pressure on the domain $x_{0} \leq x \leq 1,-1 / 2 \leq z \leq 1 / 2$ as

$$
\begin{aligned}
p(x, z, \epsilon)= & 1+\epsilon \tilde{P}_{L 1}\left(\left(x-x_{0}\right) / \epsilon, z\right)+\epsilon^{2}\left[\tilde{p}_{2}(x, z)+\tilde{v}_{2}\left(\left(x-x_{0}\right) / \epsilon, z\right)\right. \\
& \left.+\tilde{w}_{2}((1-x) / \epsilon, z)\right]+O\left(\epsilon^{3}\right) .
\end{aligned}
$$


In the above, tildes denote quantities in the trailing bearing section. The function $\tilde{p}_{2}(x, z)$ is again given by the formula (3.2), but now, values of $h(x, z)$ are those relevant to the trailing section. The function $\tilde{P}_{L 1}(\tilde{\xi}, z)$ has the representation

$$
\tilde{P}_{L 1}(\tilde{\xi}, z)=\sum_{n=1}^{\infty} k_{n} \theta_{n}(z) e^{-\tilde{\xi} \sqrt{v_{n}}}, \quad-1 / 2<z<1 / 2, \tilde{\xi} \geq 0,
$$

where $k_{n}$ are the Fourier coefficients with representation of the form (3.15), and $v_{n}$ and $\theta_{n}$ are the eigenvalues and eigenfunctions corresponding to the regular Sturm-Liouville system (3.16), (3.17) analogous to that for $\kappa_{n}$ and $\chi_{n}$, but with $h\left(x_{0}-, z\right)$ replaced by $h\left(x_{0}+, z\right)$. Similarly, $\tilde{v}_{2}(\tilde{\xi}, z)$ is the solution of a BVP analogous to $(3.18)-(3.20)$, with $h\left(x_{0}+, z\right), \tilde{\xi}=-\bar{\eta}, \tilde{p}_{2}, \tilde{P}_{L 1}(\tilde{\xi}, z)$ replacing $h\left(x_{0}-, z\right), \bar{\eta}, \bar{p}_{2}, \bar{P}_{T 1}(\bar{\eta}, z)$ respectively, and has the series representation

$$
\tilde{v}_{2}(\tilde{\xi}, z)=\sum_{n=1}^{\infty} \tilde{v}_{2 n}(\tilde{\xi}) \theta_{n}(z)
$$

where the $\tilde{v}_{2 n}$ are the solutions of the BVPs

$$
\begin{aligned}
\tilde{v}_{2 n}^{\prime \prime}(\tilde{\xi})-v_{n} \tilde{v}_{2 n}(\tilde{\xi}) & =g_{n}, \\
\tilde{v}_{2 n}(0) & =b_{n}, \\
\tilde{v}_{2 n}(\infty) & =0,
\end{aligned}
$$

for $n=1,2,3, \ldots$, while the $b_{n}$ and $g_{n}$ are the analogues of the $a_{n}$ and $\gamma_{n}$ of (3.29) and (3.25) respectively, under the above transformations.

Finally, the function $\tilde{w}_{2}(\tilde{\eta}, z)$ is given by the analogue of $(3.5)-(3.8)$, with $h(1, z)$, $\tilde{p}_{2}(1, z), \tilde{\eta}=(1-x) / \epsilon, \psi_{n}, \mu_{n}$ and $d_{n}$ replacing $h(0, z), \bar{p}_{2}(0, z), \bar{\xi}=x / \epsilon, \phi_{n}, \lambda_{n}$ and $c_{n}$ respectively.

Formulas (3.30) and (4.1) with formulas (3.2) for $\tilde{p}_{2}$, (3.14), (4.2) for $\bar{P}_{T_{1}}, \tilde{P}_{L 1}$, (3.5) for $\bar{v}_{2}, \bar{v}_{2}$ and (3.24) for $\bar{w}_{2}, \bar{w}_{2}$, provide a straightforward procedure for calculating the pressure distribution in a step slider bearing. Note the additional $O(\epsilon)$ terms in the pressure expansions (3.30) and (4.1) that appear due to the step at $x=x_{0}$. Furthermore, $\bar{P}_{T 1}$ and $\bar{P}_{L 1}$ contribute to the overall expressions for $\bar{w}_{2}$ and $\tilde{v}_{2}$ respectively, via (3.21), but, to determine these quantities, $\lambda_{1}$ and $\lambda_{2}$ need to be found. In principle, the condition (2.5) provides the required equations for this calculation, but the analysis is difficult, and can only be completed under appropriate simplifying assumptions regarding the form of the film profile function $h(x, z)$. Thus, in what follows, we restrict attention to the case where $h(x, z)=h(x)$, that is, the bearing profile only varies longitudinally. Such a bearing is termed a wedge bearing, and in the present context, the existence of a jump discontinuity at $x=x_{0}$ means that the step size, $\Delta h\left(x_{0}\right)=h\left(x_{0}+\right)-h\left(x_{0}-\right)$, is nonzero (and finite). 


\section{Construction of the function $\lambda(z, \epsilon)$}

For the wedge step bearing, the system (3.6), (3.7) is particularly simple, and the eigenfunctions $\phi_{n}(z)$ are readily shown to be

$$
\phi_{n}(z)= \begin{cases}\sqrt{\frac{2}{h^{3}(0)}} \cos (n \pi z) & n=1,3,5, \ldots, \\ \sqrt{\frac{2}{h^{3}(0)}} \sin (n \pi z) & n=2,4,6, \ldots,\end{cases}
$$

with eigenvalues $\lambda_{n}=n^{2} \pi^{2}$.

The eigenfunctions $\chi_{n}((3.16),(3.17)), \theta_{n}$ and $\psi_{n}$ are then easily shown to be given by (5.1), with $h(0)$ replaced by $h\left(x_{0}-\right), h\left(x_{0}+\right)$ and $h(1)$ respectively, while

$$
\lambda_{n}=\kappa_{n}=v_{n}=\mu_{n}=n^{2} \pi^{2}, \quad n=1,2,3, \ldots
$$

Since the pressure, as represented by (3.30) and (4.1) in the leading and trailing sections of the bearing respectively, is assumed continuous at the step $x=x_{0}$, it remains to apply the mass flow condition (2.5) to determine $\lambda_{1}, \lambda_{2}$.

Thus, substituting (3.30) and (4.1) into condition (2.5), eliminating the derivatives of $\tilde{w}_{2}$ and $\bar{v}_{2}$ at $x=x_{0} \pm$ which are exponentially small there as $\epsilon \rightarrow 0$, and equating like terms, we obtain

$$
\begin{aligned}
\left.h^{3}\left(x_{0}+, z\right) \epsilon^{2} \frac{\partial \tilde{p}_{2}}{\partial x}\right|_{x=x_{0}+}-\left.h^{3}\left(x_{0}-, z\right) \epsilon^{2} \frac{\partial \bar{p}_{2}}{\partial x}\right|_{x=x_{0}-} \\
=\left.h^{3}\left(x_{0}-, z\right) \epsilon \frac{\partial \bar{P}_{T 1}}{\partial x}\right|_{x=x_{0}-}-\left.h^{3}\left(x_{0}+, z\right) \epsilon \frac{\partial \tilde{P}_{L L}}{\partial x}\right|_{x=x_{0}+} \\
\quad+\left.h^{3}\left(x_{0}-, z\right) \epsilon^{2} \frac{\partial \bar{w}_{2}}{\partial x}\right|_{x=x_{0}-}-\left.h^{3}\left(x_{0}+, z\right) \epsilon^{2} \frac{\partial \tilde{v}_{2}}{\partial x}\right|_{x=x_{0}+} \\
\quad-\Lambda\left[h\left(x_{0}-, z\right)-h\left(x_{0}+, z\right)\right] .
\end{aligned}
$$

Substituting the definitions of $\bar{p}_{2}, \tilde{p}_{2}, \bar{P}_{T 1}, \tilde{P}_{L 1}, \bar{w}_{2}$ and $\tilde{v}_{2}$ into (5.2) and equating like powers of $\epsilon$ we obtain the following expressions:

$$
h^{3}\left(x_{0}-\right) \sum_{n=1}^{\infty} \sqrt{\kappa_{n}} j_{n} \chi_{n}(z)+h^{3}\left(x_{0}+\right) \sum_{n=1}^{\infty} \sqrt{\nu_{n}} k_{n} \theta_{n}(z)=\Lambda\left[h\left(x_{0}-\right)-h\left(x_{0}+\right)\right]
$$

and

$$
h^{3}\left(x_{0}-\right) \sum_{n=1}^{\infty} \sqrt{\kappa_{n}} a_{n} \chi_{n}(z)+h^{3}\left(x_{0}+\right) \sum_{n=1}^{\infty} \sqrt{\nu_{n}} b_{n} \theta_{n}(z)=0 .
$$


Consider now the expression (5.3). The right-hand side is a constant, hence an even function in $z$, so that from the form of the eigenfunctions $\chi_{n}(z)$ and $\theta_{n}(z)$, we deduce that $j_{n}=k_{n}=0, n=2,4,6, \ldots$ For $n=1,3,5, \ldots,(5.3)$ becomes

$$
\begin{aligned}
& \sum_{n=1,3.5 \ldots}^{\infty} n \pi\left\{\int_{-1 / 2}^{1 / 2} \lambda_{1}(z) \sqrt{2} \cos (n \pi z) d z\right\} \sqrt{2} \cos (n \pi z) \\
& =\frac{-\Lambda \Delta h\left(x_{0}\right)}{h^{3}\left(x_{0}-\right)+h^{3}\left(x_{0}+\right)} .
\end{aligned}
$$

Denoting the Fourier cosine coefficient of $\lambda_{1}(z)$ by

$$
F_{c n}\left(\lambda_{1}(z)\right)=\int_{-1 / 2}^{1 / 2} \lambda_{1}(z) \sqrt{2} \cos (n \pi z) d z, \quad n=1,3,5, \ldots,
$$

we can write (5.5) as

$$
\sum_{n=1,3,5 \ldots}^{\infty} n \pi F_{c n}\left(\lambda_{1}(z)\right) \sqrt{2} \cos (n \pi z)=-\frac{\Lambda \Delta h\left(x_{0}\right)}{h^{3}\left(x_{0}-\right)+h^{3}\left(x_{0}+\right)},
$$

from which we obtain a Fourier representation for $\lambda_{1}(z)$ as

$$
\lambda_{1}(z)=\frac{-\Lambda \Delta h\left(x_{0}\right)}{h^{3}\left(x_{0}-\right)+h^{3}\left(x_{0}+\right)} \sum_{n=1,3,5, \ldots}^{\infty} \frac{4 \sin (n \pi / 2)}{n^{2} \pi^{2}} \cos (n \pi z) .
$$

Similarly, consideration of the expression (5.4) leads to

$$
\lambda_{2}(z)=\frac{h^{3}\left(x_{0}-\right) \bar{p}_{2}\left(x_{0}-, z\right)+h^{3}\left(x_{0}+\right) \tilde{p}_{2}\left(x_{0}+, z\right)}{h^{3}\left(x_{0}-\right)+h^{3}\left(x_{0}+\right)} .
$$

Thus $\lambda(z, \epsilon)$ is given by $\lambda(z, \epsilon)=1+\epsilon \lambda_{1}(z)+\epsilon^{2} \lambda_{2}(z)+O\left(\epsilon^{3}\right)$, where $\lambda_{1}(z)$, $\lambda_{2}(z)$ are given by (5.6), (5.7) respectively. This defines the value of $\lambda(z, \epsilon)$ in terms of the values of $h(x), \bar{p}_{2}$ and $\bar{p}_{2}$ at the step.

\section{The pressure field}

Now, since $h=h(x)$ only, (3.21) becomes

$$
\begin{aligned}
L_{0}^{-} \bar{w}_{2}= & -h^{3}\left(x_{0}-\right)\left\{\left(\frac{\partial \bar{P}_{T 1}}{\partial \bar{\eta}}\right)^{2}+\bar{P}_{T 1}\left(\frac{\partial^{2} \bar{P}_{T 1}}{\partial \bar{\eta}^{2}}\right)\right\} \\
& +3 h^{2}\left(x_{0}-\right) h^{\prime}\left(x_{0}-\right)\left(\frac{\partial \bar{P}_{T 1}}{\partial \bar{\eta}}+\bar{\eta} \frac{\partial^{2} \bar{P}_{T 1}}{\partial \bar{\eta}^{2}}\right)
\end{aligned}
$$




$$
\begin{aligned}
& -h^{3}\left(x_{0}-\right)\left\{\left(\frac{\partial \bar{P}_{T 1}}{\partial z}\right)^{2}+\bar{P}_{T 1}\left(\frac{\partial^{2} \bar{P}_{T 1}}{\partial z^{2}}\right)\right\} \\
& +3 \bar{\eta} h^{2}\left(x_{0}-\right) h^{\prime}\left(x_{0}-\right) \frac{\partial^{2} \bar{P}_{T 1}}{\partial z^{2}}+\Lambda h\left(x_{0}-\right) \frac{\partial \bar{P}_{T 1}}{\partial \bar{\eta}}
\end{aligned}
$$

Moreover, for the wedge bearing, we have, from (3.11),

$$
\frac{\partial^{2} \bar{P}_{T 1}}{\partial \bar{\eta}^{2}}+\frac{\partial^{2} \bar{P}_{T 1}}{\partial z^{2}}=0
$$

so (6.1) becomes

$$
\begin{aligned}
L_{0}^{-} \bar{w}_{2}= & -h^{3}\left(x_{0}-\right)\left\{\left(\frac{\partial \bar{P}_{T 1}}{\partial \bar{\eta}}\right)^{2}+\bar{P}_{T 1}\left(\frac{\partial^{2} \bar{P}_{T 1}}{\partial \bar{\eta}^{2}}\right)\right\} \\
& -h^{3}\left(x_{0}-\right)\left\{\left(\frac{\partial \bar{P}_{T 1}}{\partial z}\right)^{2}+\bar{P}_{T 1}\left(\frac{\partial^{2} \bar{P}_{T 1}}{\partial z^{2}}\right)\right\} \\
& +3 h^{2}\left(x_{0}-\right) h^{\prime}\left(x_{0}-\right) \frac{\partial \bar{P}_{T 1}}{\partial \bar{\eta}}+\Lambda h\left(x_{0}-\right) \frac{\partial \bar{P}_{T 1}}{\partial \bar{\eta}} .
\end{aligned}
$$

To calculate $\bar{w}_{2}$, we need to solve (6.2), subject to the boundary conditions (3.22), (3.23). The series representation (3.14) of $\bar{P}_{T 1}(\bar{\eta}, z)$ makes the calculation of the right-hand side of (6.2) and the subsequent solution process calculations of extreme complexity. However, we note from (3.14), (3.15) and (5.6) that $\left|\bar{P}_{T 1}(\bar{\eta}, z)\right|$ is proportional to $\left|\Delta h\left(x_{0}\right)\right|$, and thus is small when $\Delta h\left(x_{0}\right)$ is small. Thus, for a small step, we may write (6.2) as

$$
L_{0}^{-} \bar{w}_{2}=\left[3 h\left(x_{0}-\right) h^{\prime}\left(x_{0}-\right)+\Lambda\right] h\left(x_{0}-\right) \frac{\partial \bar{P}_{T 1}}{\partial \bar{\eta}}+O\left(\left(\Delta h\left(x_{0}\right)\right)^{2}\right) .
$$

Explicitly, we have

$$
\bar{P}_{T 1}(\bar{\eta}, z)=\frac{-4 \Lambda \Delta h\left(x_{0}\right)}{h^{3}\left(x_{0}-\right)+h^{3}\left(x_{0}+\right)} \sum_{n=1,3,5, \ldots}^{\infty} \frac{\sin (n \pi / 2)}{n^{2} \pi^{2}} \cos (n \pi z) e^{-\bar{\eta}(n \pi)},
$$

so that (6.3) becomes

$$
\begin{aligned}
L_{0}^{-} \bar{w}_{2}= & \frac{4 \Lambda\left[3 h\left(x_{0}-\right) h^{\prime}\left(x_{0}-\right)+\Lambda\right] h\left(x_{0}-\right) \Delta h\left(x_{0}\right)}{h^{3}\left(x_{0}-\right)+h^{3}\left(x_{0}+\right)} \\
& \times \sum_{n=1,3,5, \ldots}^{\infty} \frac{\sin (n \pi / 2)}{n \pi} \cos (n \pi z) e^{-\bar{\eta}(n \pi)}+O\left(\left(\Delta h\left(x_{0}\right)\right)^{2}\right) .
\end{aligned}
$$


Thus, in the present case of the wedge bearing, we have

$$
\begin{aligned}
f(\bar{\eta}, z)= & \frac{4 \Lambda\left[3 h\left(x_{0}-\right) h^{\prime}\left(x_{0}-\right)+\Lambda\right] h\left(x_{0}-\right) \Delta h\left(x_{0}\right)}{h^{3}\left(x_{0}-\right)+h^{3}\left(x_{0}+\right)} \\
& \times \sum_{n=1,3,5, \ldots}^{\infty} \frac{\sin (n \pi / 2)}{n \pi} \cos (n \pi z) e^{-\bar{\eta}(n \pi)}+O\left(\left(\Delta h\left(x_{0}\right)\right)^{2}\right) .
\end{aligned}
$$

If we neglect $O\left(\left(\Delta h\left(x_{0}\right)\right)^{2}\right)$ terms in (6.4) and apply this approximate $f$ to the initial value problem (3.26)-(3.28), we obtain approximate $\bar{w}_{2 n}^{*}(\bar{\eta})$ as:

$$
\bar{w}_{2 n}^{*}(\bar{\eta})=\left(a_{n}-\frac{\bar{D}_{n} \bar{\eta}}{2 n \pi}\right) e^{-\sqrt{\kappa_{n} \bar{\eta}}}
$$

where $\bar{D}_{n}$ defines the constant

$$
\bar{D}_{n}=\frac{2 \sqrt{2} \Lambda\left[3 h\left(x_{0}-\right) h^{\prime}\left(x_{0}-\right)+\Lambda\right] h^{1 / 2}\left(x_{0}-\right) \Delta h\left(x_{0}\right)}{h^{3}\left(x_{0}-\right)+h^{3}\left(x_{0}+\right)} \frac{\sin (n \pi / 2)}{n \pi}, \quad n=1,3, \ldots
$$

Also, from (3.29), $a_{n}$ is given by

$$
a_{n}=-\frac{2 \sqrt{2} \Lambda\left[h^{\prime}\left(x_{0}+\right) h^{3}\left(x_{0}-\right)-h^{\prime}\left(x_{0}-\right) h^{3}\left(x_{0}+\right)\right] h^{-3 / 2}\left(x_{0}-\right)}{h^{3}\left(x_{0}-\right)+h^{3}\left(x_{0}+\right)} \frac{\sin (n \pi / 2)}{n^{3} \pi^{3}},
$$

for $n=1,3,5, \ldots$, so that an approximate value for $\bar{w}_{2}, \bar{w}_{2}^{*}$ is given by

$$
\bar{w}_{2}^{*}(\bar{\eta}, z)=\sum_{n=1,3,5, \ldots}^{\infty}\left(a_{n}-\frac{\bar{D}_{n} \bar{\eta}}{2 n \pi}\right) e^{-\sqrt{\kappa_{n} \bar{\eta}}} \chi_{n}(z) .
$$

Similarly, by replacing $h^{3}\left(x_{0}-, z\right), a_{n}, \bar{D}_{n}, \kappa_{n}, \chi_{n}$ and $\bar{\eta}$ with $h^{3}\left(x_{0}+, z\right), b_{n}, \tilde{D}_{n}, v_{n}$, $\theta_{n}$ and $\tilde{\xi}$ respectively throughout $(3.18)-(6.6)$ we obtain an approximate $\tilde{v}_{2}^{*}(\tilde{\xi}, z)$ as

$$
\tilde{v}_{2}^{*}(\tilde{\xi}, z)=\sum_{n=1,3,5, \ldots}^{\infty}\left(b_{n}-\frac{\tilde{D}_{n} \tilde{\xi}}{2 n \pi}\right) e^{-\sqrt{\nu_{n}} \tilde{\xi}} \theta_{n}(z)
$$

where $\tilde{\xi}=\left(x-x_{0}\right) / \epsilon$ is the boundary layer variable, and $\tilde{D}_{n}$ defines the constant

$$
\bar{D}_{n}=\frac{2 \sqrt{2} \Lambda\left[3 h\left(x_{0}+\right) h^{\prime}\left(x_{0}+\right)+\Lambda\right] h^{\frac{1}{2}}\left(x_{0}+\right) \Delta h\left(x_{0}\right)}{h^{3}\left(x_{0}-\right)+h^{3}\left(x_{0}+\right)} \frac{\sin (n \pi / 2)}{n \pi}, \quad n=1,3, \ldots,
$$

with $b_{n}$ given by, for $n=1,3,5, \ldots$,

$$
b_{n}=\frac{2 \sqrt{2} \Lambda\left[h^{\prime}\left(x_{0}+\right) h^{3}\left(x_{0}-\right)-h^{\prime}\left(x_{0}-\right) h^{3}\left(x_{0}+\right)\right] h^{-3 / 2}\left(x_{0}+\right)}{h^{3}\left(x_{0}-\right)+h^{3}\left(x_{0}+\right)} \frac{\sin (n \pi / 2)}{n^{3} \pi^{3}} .
$$


The representations (6.6), (6.7) give approximations to the functions $\bar{w}_{2}$ and $\tilde{v}_{2}$ that include terms (arising from $a_{n}, b_{n}$ ) that are $O(1)$ as the step size $\Delta h\left(x_{0}\right) \rightarrow 0$, and terms (arising from $\bar{D}_{n}, \tilde{D}_{n}$ ) that are $O\left(\Delta h\left(x_{0}\right)\right.$ ). In principle, the quadratic terms on the right-hand sides of (3.21) and (6.2) could be included to provide a higher-order correction. This has not been done here.

To complete the construction of approximations to the pressure field in the bearing, we note that since $h$ is a function of $x$ only, that is, $h=h(x)$, the functions $\bar{p}_{2}, \tilde{p}_{2}$ are given by

$$
\bar{p}_{2}(x, z)=\tilde{p}_{2}(x, z)=\frac{1}{2} \Lambda h^{\prime}(x) h^{-3}(x)\left(z^{2}-1 / 4\right),
$$

where the values of $h(x)$ and $h^{\prime}(x)$ are assumed to be those appropriate to the regions before and after the jump discontinuity at $x=x_{0}$.

Thus the pressure distribution over the whole bearing domain $0 \leq x \leq 1,-1 / 2 \leq$ $z \leq 1 / 2$ is given approximately by

$$
\begin{aligned}
p(x, z, \epsilon)= & 1+\Lambda \epsilon\left\{\left[4-2 h^{-2}\left(x_{0}-\right)\left[3 h\left(x_{0}-\right) h^{\prime}\left(x_{0}-\right)+\Lambda\right]\left(x_{0}-x\right)\right]\right. \\
& \times C_{1} G_{2}\left(x_{0}-x, z, \epsilon\right) \\
& +\epsilon\left[\bar{p}_{2}(x, z)+4 h^{\prime}(0) h^{-3}(0) G_{3}(x, z, \epsilon)\right. \\
& \left.\left.-4 h^{-3}\left(x_{0}-\right) C_{2} G_{3}\left(x_{0}-x, z, \epsilon\right)\right]\right\}+O\left(\epsilon\left(\Delta h\left(x_{0}\right)\right)^{2}\right)
\end{aligned}
$$

on $0 \leq x \leq x_{0}$, and

$$
\begin{aligned}
p(x, z, \epsilon)= & 1+\Lambda \epsilon\left\{\left[4-2 h^{-2}\left(x_{0}+\right)\left[3 h\left(x_{0}+\right) h^{\prime}\left(x_{0}+\right)+\Lambda\right]\left(x-x_{0}\right)\right]\right. \\
& \times C_{1} G_{2}\left(x-x_{0}+, z, \epsilon\right) \\
& +\epsilon\left[\tilde{p}_{2}(x, z)+4 h^{\prime}(1) h^{-3}(1) G_{3}(1-x, z, \epsilon)\right. \\
& \left.\left.+4 h^{-3}\left(x_{0}+\right) C_{2} G_{3}\left(x-x_{0}+, z, \epsilon\right)\right]\right\}+O\left(\epsilon\left(\Delta h\left(x_{0}\right)\right)^{2}\right)
\end{aligned}
$$

on $x_{0} \leq x \leq 1$, where

$$
\begin{aligned}
& C_{1}=-\frac{\Delta h\left(x_{0}\right)}{h^{3}\left(x_{0}-\right)+h^{3}\left(x_{0}+\right)}, \\
& C_{2}=\frac{h^{\prime}\left(x_{0}+\right) h^{3}\left(x_{0}-\right)-h^{\prime}\left(x_{0}-\right) h^{3}\left(x_{0}+\right)}{h^{3}\left(x_{0}-\right)+h^{3}\left(x_{0}+\right)},
\end{aligned}
$$

while $G_{p}(t, z, \epsilon)$ is given by

$$
G_{p}(t, z, \epsilon)=\sum_{m=0}^{\infty} \frac{(-1)^{m}}{(2 m+1)^{p} \pi^{p}} \cos (2 m+1) \pi z e^{-(2 m+1) \pi t / \epsilon}
$$




\section{The load}

The non-dimensional load, $W$, for the general bearing may be defined as

$$
W=\int_{-1 / 2}^{1 / 2} \int_{0}^{1}(p(x, z, \epsilon)-1) d x d z
$$

where $p(x, z, \epsilon)$ is the pressure. Thus $W$ is the excess pressure over the ambient, summed over the bearing.

If the expansions (6.8), (6.9) are applied to (7.1), we obtain, after some calculation, the non-dimensional load, $W$, over the domain $0 \leq x \leq 1,-1 / 2 \leq z \leq 1 / 2$ as

$$
W=\epsilon^{2} \Lambda\left(W_{0}+\epsilon W_{1}\right)+O\left(\epsilon^{2}\left(\Delta h\left(x_{0}\right)\right)^{2}\right)
$$

where

$$
W_{0}=\frac{1}{24}\left[h^{-2}(1)-h^{-2}\left(x_{0}+\right)+h^{-2}\left(x_{0}-\right)-h^{-2}(0)\right]-\frac{16}{\pi^{4}} C_{1} S_{4}
$$

and

$$
\begin{aligned}
W_{1}= & -\frac{4}{\pi^{5}} C_{1}\left[h^{-2}\left(x_{0}-\right)\left[3 h\left(x_{0}-\right) h^{\prime}\left(x_{0}-\right)+\Lambda\right]\right. \\
& \left.+h^{-2}\left(x_{0}+\right)\left[3 h\left(x_{0}+\right) h^{\prime}\left(x_{0}+\right)+\Lambda\right]\right] S_{5} \\
& +\frac{8}{\pi^{5}}\left[h^{-3}(0) h^{\prime}(0)+h^{-3}(1) h^{\prime}(1)+C_{2}\left(h^{-3}\left(x_{0}+\right)-h^{-3}\left(x_{0}-\right)\right)\right] S_{5} \\
& +O\left(\epsilon^{2}\left(\Delta h\left(x_{0}\right)\right)^{2}\right),
\end{aligned}
$$

where $S_{n}=\sum_{m=0}^{\infty}(2 m+1)^{-n}$.

\section{Discussion}

The expressions (6.8), (6.9) for the pressure field, and (7.2) for the load provide explicit readily computed approximations valid to the extent indicated for a range of wedge-bearing profiles $h(x)$, provided $\epsilon$ and $\Delta h\left(x_{0}\right)$ are small. Note that in (6.8), (6.9), the $O(\epsilon)$ contributions vanish if $\Delta h\left(x_{0}\right)=0$, that is, there is no step; while terms $O\left(\epsilon^{2}\right)$ still include layer terms at $x=0, x_{0}$ and 1 , arising from the imposition of ambient pressure at $x=0,1$, and the change in slope, $\Delta h^{\prime}\left(x_{0}\right)$, of the profile at $x=x_{0}$. In fact, if $h(x)$ is continuous at $x=x_{0},(6.10),(6.11)$ give

$$
C_{1}=0, \quad C_{2}=\frac{1}{2}\left(h^{\prime}\left(x_{0}+\right)-h^{\prime}\left(x_{0}-\right)\right)=\frac{1}{2} \Delta h^{\prime}\left(x_{0}\right),
$$

and (6.8), (6.9) reduce to those obtained in [4]. If $h(x)$ is smooth at $x=x_{0}$, so that $\Delta h^{\prime}\left(x_{0}\right)=0, C_{2}=0$ also, and $(6.8),(6.9)$ reduce to those obtained in [7], with layers only at $x=0,1$. 


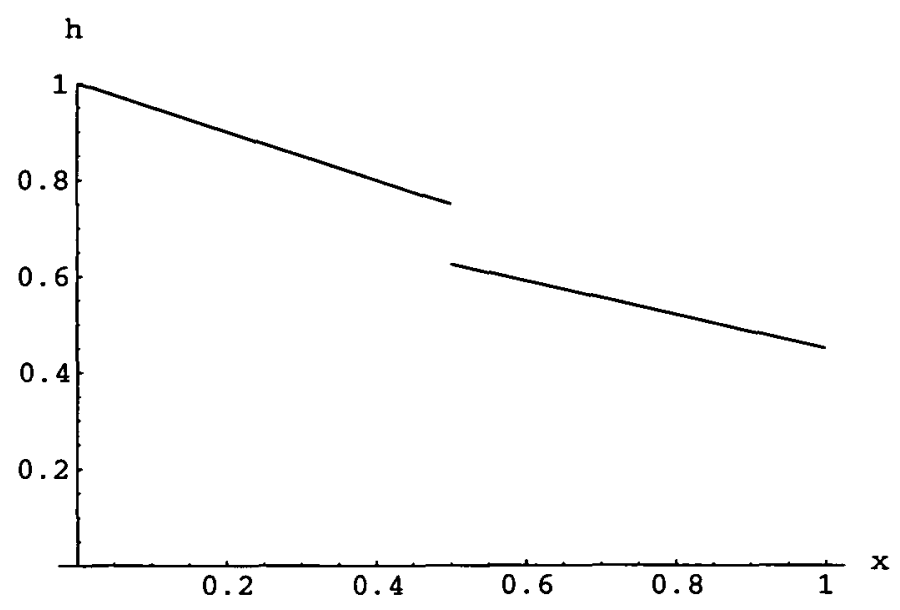

FIGURE 2. Wedge step bearing profile: $l_{1}(x)=1-0.5 x, l_{2}(x)=0.8-0.35 x$ with $x_{0}=1 / 2$.

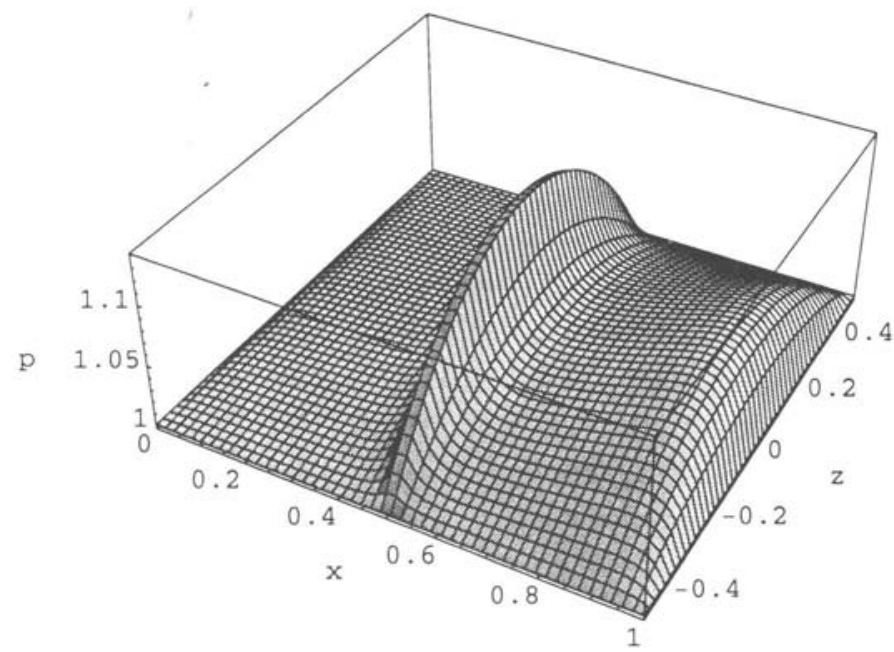

FIGURE 3. Surface plot of non-dimensional pressure for the wedge step slider bearing as given by asymptotic formulae (6.8) and (6.9) for the linear bearing profiles $l_{1}(x)=1-0.5 x, l_{2}(x)=0.8-0.35 x$, with $\epsilon=0.1, \Lambda=20$ and $x_{0}=1 / 2$. 


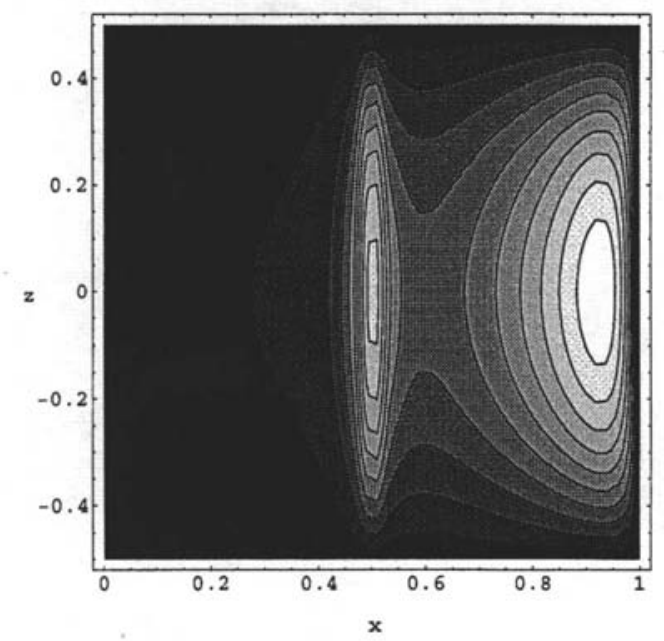

FIGURE 4. Contour plot of non-dimensional pressure for the wedge step bearing as given by asymptotic formulae (6.8) and (6.9) for the linear bearing profiles $l_{1}(x)=1-0.5 x, l_{2}(x)=0.8-0.35 x$, with $\epsilon=0.1, \Lambda=20$ and $x_{0}=1 / 2$.

Similar comments can be made regarding the load expressions (7.2)-(7.4). In (7.2), contributions are made from both step and slope change at the $O\left(\epsilon^{2}\right)$ and $O\left(\epsilon^{3}\right)$ levels. If there is no step, $C_{1}=0$, and the $C_{2}$ contribution arising only in (7.4) vanishes, so that there is no contribution (at the level considered) of any change of slope at $x=x_{0}$. This is consistent with the findings of [4], and in fact, the load formula (7.2) reduces to that found in [4]. On the other hand, even if $\Delta h^{\prime}\left(x_{0}\right)=0$, any finite step contributes at both the $O\left(\epsilon^{2}\right)$ and $O\left(\epsilon^{3}\right)$ level, through $C_{1}$. Furthermore, if the bearing profile is overall converging (as is experienced in practice), so that $\Delta h\left(x_{0}\right)<0$, we have $C_{1}>0$, and the step acts to increase the load-bearing properties of the bearing.

Figure 2 displays a simple linear wedge-bearing profile, given by

$$
h(x, z)= \begin{cases}1-0.5 x, & 0 \leq x \leq 1 / 2, \\ 0.8-0.35 x, & 1 / 2 \leq x \leq 1,\end{cases}
$$

for which $x_{0}=0.5, \Delta h\left(x_{0}\right)=-0.125$ and $\Delta h^{\prime}\left(x_{0}\right)=0.15$. The corresponding (approximate) pressure distribution as given by (6.8), (6.9) is displayed in the threedimensional plot of Figure 3. The layers at the step $x=0.5$ and trailing edge $x=1$ are clearly in evidence; the layer at the leading edge $x=0$ is more subtle. Figure 4 shows the contour plot of this, emphasising the layer structure. Figure 5 compares longitudinal plots of the pressure as given by (6.8), (6.9) along the bearing midline $z=0$ with those obtained using a numerical package [3], for small values of $\epsilon$. The accuracy of the asymptotic formulae clearly increases as $\epsilon \rightarrow 0$. 

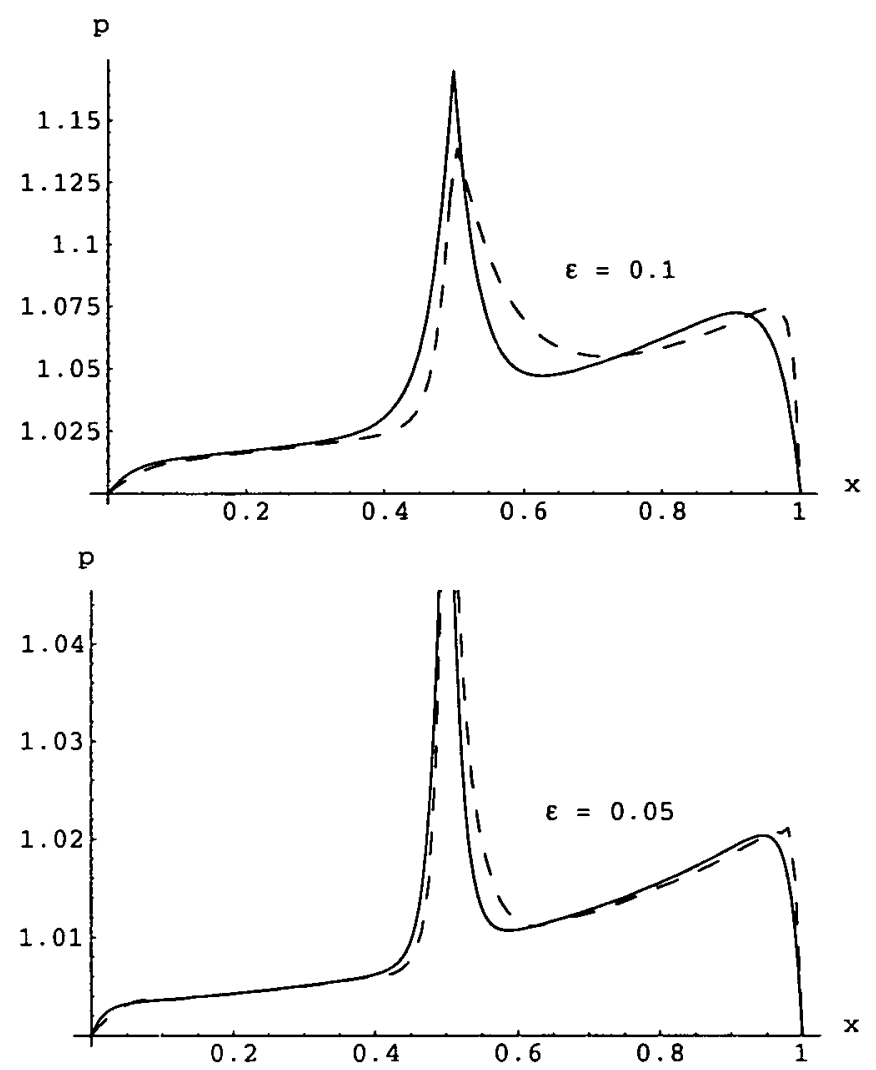

FIGURE 5. Comparison of one-dimensional plots of the pressure for the wedge step bearing as given by asymptotic formulae (6.8) and (6.9) (solid line) with that obtained using PDEase ([3]) (dashed line), for the bearing profiles $l_{1}(x)=1-0.5 x, l_{2}(x)=0.8-0.35 x$, with $\Lambda=20, x_{0}=1 / 2$ and varying $\epsilon$.

\section{Acknowledgements}

The authors wish to thank the referees for a number of useful comments.

\section{References}

[1] W. Boyce and R. C. DiPrima, Elementary differential equations and boundary value problems, 5th ed. (Wiley, New York, 1992).

[2] R. C. DiPrima, "Asymptotic methods for an infinitely long step slider squeeze bearing", J. Lub. Tech., Trans. ASME 95 (1973) 208-215.

[3] Macsyma, Inc., PDEase 2, Finite element analysis for partial differential equations, (Macsyma, Inc., Arlington, 1993-1996). 
[4] I. Penesis, J. J. Shepherd and H. J. Connell, "Asymptotic analysis of narrow gas-lubricated slider bearings with non-smooth profiles", in Proceedings of EMAC98, 3rd Biennial Engineering Mathematics and Applications Conference, (The Institution of Engineers, Adelaide, Australia, 1998) 163-166.

[5] J. Penesis, J. J. Shepherd and H. J. Connell, "The pressure field in a two-dimensional taper-taper gas-lubricated bearing of narrow geometry", in EMAC 2000 Proceedings, 4rd Biennial Engineering Mathematics and Applications Conference, (The Institution of Engineers, Melbourne, Australia, 2000) 239-242.

[6] J. A. Schmitt and R. C. DiPrima, "Asymptotic methods for an infinite slider bearing with a discontinuity in film slope", J. Lub. Tech., Trans. ASME 98 (1976) 446-452.

[7] J. J. Shepherd and R. C. DiPrima, "Asymptotic analysis of a finite gas slider bearing of narrow geometry", J. Lub. Tech., Trans. ASME 105 (1983) 491-495.

[8] S. Wolfram, Mathematica: a system for doing mathematics by computer, 2nd ed. (Addison-Wesley, Redwood City, CA, 1991). 